\title{
Triticale-wheat hybrid lines with the vaviloid type of spike branching
}

Adonina I.G. ${ }^{1}$, Mehdiyeva S.P. ${ }^{2}$, Prokopjeva M.V. ${ }^{3 *}$, Aminov N.Kh. ${ }^{2}$, Salina E.A. ${ }^{1}$

${ }^{1}$ Institute of Cytology and Genetics, SB RAS, Novosibirsk, Russia

${ }^{2}$ Genetic Resources Institute, ANAS, Baku, Azerbaijan

${ }^{3}$ Novosibirsk State University, Novosibirsk, Russia

*e-mail:prokopjeva.marjana@yandex.ru

The collection of wheat forms with the vaviloid type of spike branching consists of lines obtained from crossing a stable wheat-rye amphiploid (triticale) ABR $(2 n=6 x=42)$, used as the maternal form with the local variety of common wheat Triticum aestivum var. velitinum $(2 n=6 x=42$, BAD). Triticale was obtained by professor Aminov in 1975 from hybridization of synthetic wheat BAD $(T$. durum $\times$ Ae. squarrosa var. meyeri, catalog number in VIR - k-45918) with weed rye Secale cereale ssp. segetale $(2 n=2 x=14$, RR). Hybrid populations were studied for 10 years in an open field at the Absheron experimental base of the Genetic Resources Institute of ANAS. The forms with the vaviloid type of spike branching began to appear from the generation F4. Estimation of the morphological and quantitative traits of these lines was conducted. Significant differences were registered in such parameters as the number of grains per spike, the grain weight per spike. A molecular-cytological analysis of the lines was carried out using the methods of genomic in situ hybridization (GISH) and fluorescent in situ hybridization (FISH) with probes that allow the identification of wheat and rye chromosomes. It was shown that in all the studied lines, the chromosomes 2D was replaced by a pair of rye chromosomes, presumably 2R. In addition, a part of the lines contained a telocentric chromosomes, presumably corresponding to the short arm of a wheat chromosome 2D. Thus, the studied lines with the vaviloid type of spike branching can be divided into three groups: 1) lines with one telocentric chromosome; 2) lines with a pair of telocentric chromosomes; 3) lines without telocentric chromosomes.

Acknowledgements: The study has been supported by the Budget project 0324-20190039 . 